\title{
Gas Flow and Ion Transfer in Heated ESI Capillary Interfaces
}

\author{
Laurent Bernier, ${ }^{1}$ Harry Pinfold, ${ }^{2}$ Matthias Pauly, ${ }^{2,3}$ Stephan Rauschenbach,,${ }^{2,4}$ \\ Julius Reiss ${ }^{1}$ \\ ${ }^{1}$ TU Berlin, Berlin, Germany \\ ${ }^{2}$ MPI f. Solid State Research, Stuttgart, Germany \\ ${ }^{3}$ CNRS, Institut Charles Sadron, Université de Strasbourg, Strasbourg, France \\ ${ }^{4}$ Department of Chemistry, Oxford University, Oxford, UK
}

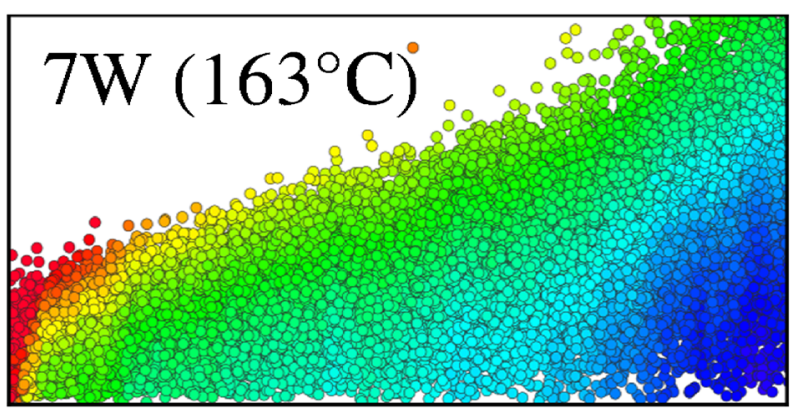

Abstract. Transfer capillaries are the preferred means to transport ions, generated by electrospray ionization, from ambient conditions to vacuum. During the transfer of ions through the narrow, long tubes into vacuum, substantial losses are typical. However, recently it was demonstrated that these losses can be avoided altogether. To understand the experimental observation and provide a general model for the ion transport, here, we investigate the ion transport through capillaries by numerical simulation of interacting ions. The simulation encompasses all relevant factors, such as space charge, diffusion, gas flow, and heating. Special attention is paid to the influence of the gas flow on the transmission and especially the change imposed by heating. The gas flow is modeled by a one-dimensional gas dynamics description. A large number of ions are treated as point particles in this gas flow. This allows to investigate the influence of the capillary heating on the gas flow and by this on the ion transport. The results are compared with experimental findings.

Keywords: ESI, Transport capillary, Atmospheric pressure interfaces, Simulation, Transfer, High transfer, Gasdynamic, Gasflow, Design principles

Received: 29 November 2016/Revised: 10 January 2018/Accepted: 16 January 2018/Published Online: 21 February 2018

\section{Introduction}

A tmospheric pressure interfaces (API) link vacuum-based analytical and preparation technologies with ambient soft ionization mechanisms like electrospray ionization (ESI), which provides intact molecular gas phase ions from nonvolatile species in solution [1-6]. Most importantly, mass spectrometry uses ESI-API to analyze complex organic molecules.

Ions generated at ambient conditions are usually transferred to vacuum through a narrow capillary or an orifice. Generally, this transfer is accompanied by losses, often very high in the range above $90 \%$ of the introduced ion current $[5,7]$. Recently,

Electronic supplementary material The online version of this article (https:// doi.org/10.1007/s13361-018-1895-0) contains supplementary material, which is available to authorized users.

Correspondence to: Julius Reiss; e-mail: reiss@tnt.tu-berlin.de several experiments demonstrated significantly enhanced transfer efficiency for capillaries with relatively simple modifications of the geometry [8-11]. Unity transmission was shown for a wide range of practical parameters using nanoelectrospray sources and capillaries with funnel-shaped inlet geometry [8], and for inverse funnel-shaped inlets [9]. These achievements were empirically reached but nevertheless show the great potential for performance improvements and furthermore raise the question under which circumstances highefficiency vacuum transfer is possible.

The transport of ions is governed by electric forces, externally applied and from space charge of the ion cloud, as well as their interaction with the flowing background gas via diffusion and drag $[7,8]$. Apart from the influence of a given geometry, additional circumstances such as the heating of the capillary, the overall pressure gradient, friction or the parameters of the electrospray can be influential. The transfer efficiency of a capillary interface is the result of the interplay of all these effects, which renders a comprehensive theoretical description 
of the seemingly simple atmospheric pressure-vacuum interface a very complex problem.

The transport of ions in the gas flow of an API is remarkably little studied despite its pivotal role in mass spectrometry. Only some specific aspects such as special inlet geometries in [8] or the coupling of the emerging jet to ion optics in [12] were successfully investigated and showed improvements of the transmission rate. Other works focus on specific aspects of the problem [13-20]. Specifically, Ref. [17] contains a discussion on gas flow simulations in a high pressure ion funnel combined with vacuum transfer devices of various shapes. In [18], gas flows in a complex configuration utilizing skimmers are presented. The effects of the heating on the gas flow are also investigated in [19]. Two recent articles, [20] and [21], present comparisons between flow simulations and experimental data. Remarkably, and despite comparable dimensions, the first article finds a turbulent flow, while the second one finds agreement with a laminar flow. This underlines the need for a profound understanding of the involved processes for the development of ion sources of improved performance.

One major comprehensive study, that combines experimental and theoretical considerations for the capillary transport is found in the work of Lin and Sunner, where the gas flowdriven ion transport in long, narrow capillaries at transmission rates in the order of a few percent is measured described [7]. The experimental performance of these capillaries was in agreement with estimates based on modeling the transport via a laminar flow of flat or parabolic profile including spacecharge effects and diffusion. While this work is able to rationalize the observations made for the considered range of geometry, high transmission rates can be expected only for short capillaries. Furthermore, even though the conditions in the considered capillaries were estimated to be turbulent, all calculations were made for a laminar flow, finding, nonetheless, good agreement.

To be able to describe the state of the gas flow and the corresponding ion transport, we here present a comprehensive model describing the charge transport in the gas flow in heated capillaries, with the design described in Ref. [8] which is used as experimental reference. We include all important transport mechanisms, which are diffusion, convection, and spacecharge-driven transport, creating a model covering the influences of the key external parameters such as capillary geometry, gas flow, heating, spray geometry, and ion species.

The relevant time scale on which particles adapt to the surrounding gas-velocities is as low as $10^{-10} \mathrm{~s}$ (see Supp. Mat. S.1). Compared to the typical convection time of the gas, $10^{-4} \mathrm{~s}$, (see Sec. 2.1), this means that the ions follow the gas flow nearly instantaneously which means that the gas flow dominates the transport. It is, therefore, of paramount importance to describe the gas flow in detail in order to understand the ion transport and losses. We particularly find that including turbulence is essential to the description of the experimental observations.

In our approach, a full, three-dimensional gas flow is modeled as a quasi one-dimensional flow approximated in three dimensions by the velocity profiles known for laminar or turbulent flow. A large number of ions are simulated as point particles moved by the drag force of the flowing gas, the force from external and space-charge electric fields, and diffusion; the latter taking into account the laminar or turbulent flow of the gas.

Due to the confined geometry and the strong heating, the gas flow can have non-trivial properties like thermal choking or turbulent transition. Based on the simulation of the ion motion, we can estimate the effects of droplet life time, space charge, and turbulence on the ion transport and finally simulate ion motion in a realistic geometry reproducing key device characteristics. The comparison of the simulation which results to data from experiments using short and wide transfer tubes shows quantitative and qualitative agreement with the observed transfer characteristics and temperature behavior, respectively.

Our results show that unity transport is possible below a certain space-charge limit defined by the capillary geometry if laminar flow can be maintained in the whole capillary. Further, we demonstrate that the injection of the charged particles into the capillary is crucial for the transmission efficiency and that the heating does not only affect droplet desolvation but also has a crucial effect on the turbulent transition and thus can be significant for the overall losses. Our results further show that the one-dimensional modeling of the gas flow allows to describe the basic transport in a simple cylindrical tube geometry, which leads to design rules for the layout of atmospheric interfaces. It reveals the potential for tailoring the gas flow by more sophisticated geometries, which in the future will require an in-depth treatment of the gas flow as well as the droplet desolvation to be able to understand and develop more sophisticated source designs allowing higher transmitted currents.

\section{Results}

\section{Scales and Scope of the Simulation}

The complexity of the problem makes it necessary to initially consider the relevant scales of the gas flow and the ion motion to gauge the numerical effort and the scope of the simulation. This encompasses the gas flow itself, the electrostatic interactions of the moving ions and finally the behavior of the droplets.

Estimates of the Gas Flow The gas flow is caused by the pressure difference between the first pumping stage and the ambient environment. In the straight section of the tube, the gas accelerates approximately to the local speed of sound. For an adiabatic acceleration, the gas velocity is $v_{g}=\sqrt{2 / \gamma+1} c_{0}=0.913 c_{0}$ [22]. With an ambient speed of sound $c_{0}=343 \mathrm{~m} / \mathrm{s}$, and the adiabatic index of air $\gamma=1.4$; this leads to a gas velocity of $v_{g} \approx 300 \mathrm{~m} / \mathrm{s}$. The convection (i.e., transport) time of the gas is therefore $\tau=L / v_{g} \approx 0.2 \mathrm{~ms}$ with $L=6 \mathrm{~cm}$ as the conduit's length. In contrast, the transport time of droplets in the 
region before the transfer tube (e.g., funnel-shaped part, see Figure 1) is considerably longer and noticeably depends on the spray needle position (see Supp. Mat. S.2). Further, flow velocities comparable to the speed of sound lead to compressibility effects and a strong coupling with the thermodynamics of the gas.

At this velocity, the gas volume flow is approximated as $Q_{\text {gas }}=v_{g} \pi(d / 2)^{2} \approx 1 \mathrm{~m}^{3} / \mathrm{h}$ for a capillary of diameter $d=1 \mathrm{~mm}$. The (liquid) solvent flow in the order of $30 \mu \mathrm{L} / \mathrm{h}$ translates into a 1000 times smaller gaseous volume flow upon its evaporation, by which the properties and temperature of the carrier gas are barely influenced.

However, the gas flow is heated with a power in the order of $Q=10-50 \mathrm{~W}$. The corresponding temperature evolution can be estimated to $\Delta T=200 \mathrm{~K}$ by considering the relation $c_{p} \Delta T=Q /\left(\rho \mathrm{v}_{g} A\right)$, with $c_{p}$ the specific heat capacity and $\rho$ the density of air. Clearly such a heating will significantly influence the gas properties and must therefore be considered in detail when modeling the gas flow.

The effect of wall friction is difficult to estimate by empirical formulas, since the parameters of this chocked configuration in a capillary differs from a typical large pipe flow. A simulation of a laminar flow is shown in Supp. Mat. S.3. The pressure drops by a factor of 2 in the straight part of the capillary. In principle, this effect should be considered, and the impact on the ion transport will be discussed in Sec. 2.4.2.

A crucial aspect of a gas flow is the possibility of turbulence, which significantly alters the flow pattern of the gas and hence has a strong effect on the motion of embedded ions. The dimensionless Reynolds-number is the key quantity to determine whether turbulence occurs in the considered flow. In the presented configuration (with the kinematic viscosity of air $v=$ $1.4 \times 10^{-5} \mathrm{~m}^{2} / \mathrm{s}$ ), the Reynolds-number is $\operatorname{Re}=v_{g} d / v \approx 20000$, for which turbulent flow is expected in pipes.

Turbulence is, however, a dynamic phenomenon and thus its time scale must be considered. The turn-over time of the

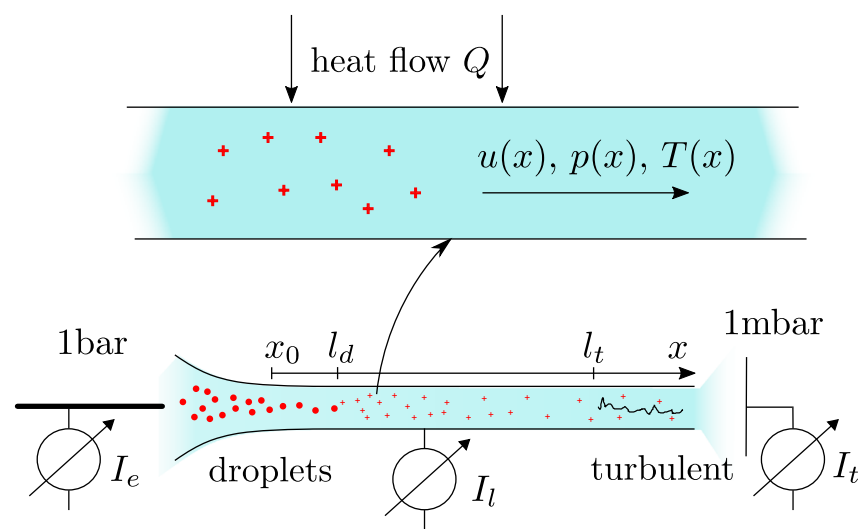

Figure 1. Sketch of the experimental setup and the coordinate system used for the modeling. The start of the straight part of the capillary defines $x_{0}=0 \mathrm{~cm}$. The model parameters are indicated: $l_{d}$ is the position where the droplet desolvation is complete, and $l_{t}$ the position where the turbulence transition occurs. Droplets are schematically represented by circles and ions by crosses smallest eddies, $\tau_{e}$, is the shortest one and is estimated from its size $\eta=\mathrm{LRe}^{-3 / 4}$, and its velocity $v_{e}=\mathrm{uRe}^{-1 / 4}$ and gives $\tau_{e}=\eta / v_{e} \approx 3 \times 10^{-8} \mathrm{~s}$ as calculated from the length of the capillary and the estimated gas velocity [23].

Both time scales describing the gas flow, convection time, and eddy turn-over time, are much longer than the particle response time. This leads to the important conclusion that the change of the ion velocity via drag effects is quasi instantaneous and, thus, the ion velocity is given as the gas velocity plus the drift velocity.

Finally, the ion movement in an interacting gas is characterized by the particle response time $\tau_{p}$, the time scale of the ion (velocity $v_{i}$ ) adopting the gas velocity $v_{g}$. This quantity can be estimated based on the properties of the ion, i.e., its mass, charge, and mobility (see Supp. Mat. S.1). The particle response time for a relatively light ion of single charge such as Rhodamine B (RhoB, $443 \mathrm{u}$ ) is $\tau_{p} \approx 4 \times 10^{-10} \mathrm{~s}$ as estimated from the mobility $\left(K_{0}=9.2 \times 10^{-5} \mathrm{~m}^{2} /(\mathrm{V} \mathrm{s})\right)$ given by the SDS package from SIMION for standard conditions [8]. This value is much shorter than the time scale of convection and even much shorter than the collision rate with the gas molecules. We thus generally assume that ions follow the gas motion instantaneously, which is also correct for heavier ions.

Estimates of the Electrostatic Interaction The ion currents in the experiment are in the range of 10-100 nA, aligned to the experimental measurement found in [8]. Compared with the moving background gas, the mass and momentum of this ion current is negligible so that feedback on the gas flow can be neglected, since the mass of the roughly $10^{18}$ gas molecules is much higher than the $10^{8}$ ions estimated from the current and convection time.

Coupled to external fields, which can occur at the inlet and exit of the tube, the exerted force over the cross section area of the tube can be considered as a pressure of approximately $2.5 \times$ $10^{-2}$ mbar (see Supp. Mat. S.1). Compared to the overall pressure difference of $1000 \mathrm{mbar}$, this effect, too, is negligible in modeling the experiment.

Droplets and Desolvation The ions in the considered experiment are the result of the droplet evolution after an electrospray [24]. In this framework, droplet size, charge state, and lifetime are the most important quantities for an estimation of the droplet behavior in the gas flow. However, they are difficult to obtain.

Following Kerbale and Tang [25], the initially created droplet is stable for a relatively long time. The processes following after a first fission are quite rapid so that the lifetime of the initial droplet becomes an approximation for the duration of the entire process, after which the gas phase ions are rapidly released.

The droplets are created in the funnel region, outside of the cylindrical conduit considered in the simulation [8]. The estimation outlined in Supp. Mat. S.2 yields the point of complete desolvation inside the first part of the cylindrical conduit, 
which is included into the simulation. Since the exact value is difficult to determine a priori, it is adapted in the simulation to fit the measurements.

\section{Experimental Setup}

The results of the modeling scheme presented in this manuscript are compared to a basic atmospheric interface using capillaries with a funnel-shaped inlet as described in Ref. [8] (see also Figure 1). A metal capillary of diameter $0.75 \mathrm{~mm}$ or $1.0 \mathrm{~mm}$ and of $6 \mathrm{~cm}$ length is mounted within an electrically isolated copper cylinder containing a cartridge heater (Watlow) with a resistive thermometer (type $\mathrm{K}$ thermocouple). This enables us to heat the capillary homogeneously and precisely control its temperature (via a calibration curve, see Supp. Mat. S.4) while at the same time the loss current $I_{\text {loss }}$ can be measured with picoampere precision on the copper cylinder. Glass capillaries are not used in the current experiment. The electrically insulating material renders the modeling of an ion-wall interaction more difficult than assuming neutralization at the wall and further lower transmission rate is reported in the literature [7].

The setup is further equipped with a low flow electrospray emitter made from pulled glass capillaries, operated at flow rates of approx. $30 \mu \mathrm{L} / \mathrm{h}$. A solution of $10^{-4} \mathrm{M}$ Rhodamine B (RhoB) in a 1:1 $\mathrm{H}_{2} \mathrm{O} / \mathrm{MeOH}$ solvent mixture is used for the transmission quantification. A high voltage is applied to this emitter to form the electrospray, while at the same time the emission current $\left(I_{\mathrm{em}}\right)$ is measured.

The vacuum chamber is pumped to a pressure of 5 mbar, which varies slightly with the temperature of the inflowing gas (see Supp. Mat. S.6). A current detector electrode, large enough to detect all transmitted charges, is mounted on the beam axis defined by the capillary exit $5 \mathrm{~cm}$ downstream and records the transmitted current $I_{\text {trans }}$. The current measurement is performed with home-build picoamperemeters based on highgain operational amplifiers, which can be floated on high voltages, and with Keithey 617 picoamperemeters/ electrometers.

The three currents are found to fulfill the condition $I_{\mathrm{em}}=I_{\text {loss }}+I_{\text {trans }}$ at every time: Many times throughout the experiments the consistency of this current measurement is tested, typically by placing the emitter at positions far off the inlet, where no transmission occurs. Then $I_{\text {trans }}=0 \mathrm{pA}$ and $I_{\mathrm{em}}=I_{\text {loss }}$ is consistently measured.

To be able to compare our modeling results with the experiments, it is important to determine which fraction of the electric heat power enters the gas flow and which is diverted into the machine. By measuring the powertemperature characteristic with and without gas flow, we determine the heat flow transfered into the gas for each temperature as the power difference between the two cases (see Supp. Mat. S.4). For the presented setup, we find that approximately one third of the electric heating power is transmitted to the gas.

\section{Gas Flow in a Heated Capillary}

One-Dimensional Description The starting point for describing the ion transport is the gas flow through the capillary. Due to the cylindrical symmetry of the ion transfer conduit, it is reasonable to describe the gas flow by a one-dimensional approximation, an approach well established in engineering, yielding good approximations for a first insight into the problem, while limiting the computational effort dramatically.

The state of the gas flow is described by the conservation laws of mass, momentum, and energy (Eqs. 1-3), modified by the heat exchange with the tube wall $[22,26]$. Assuming a steady state flow of gas (adiabatic constant $\gamma$ ) with a dominant direction this yields

$$
\begin{aligned}
& \rho_{2} u_{2}=\rho_{1} u_{1} \\
& \rho_{2} u_{2}^{2}+p_{2}=\rho_{1} u_{1}^{2}+p_{1}+\Delta q_{u} \\
& \frac{u_{2}^{2}}{2}+\frac{p_{2}}{\rho_{2}} \frac{\gamma}{\gamma-1}=\frac{u_{1}^{2}}{2}+\frac{p_{1}}{\rho_{1}} \frac{\gamma}{\gamma-1}+\Delta q .
\end{aligned}
$$

The indices 1,2 refer to two arbitrary positions in the tube $x_{1}, x_{2}$ (see Figure 1). $p$ is the pressure, $\rho$ the mass-density, and $u$ the velocity component parallel to the capillary axis.

The heat flux term $\Delta q$ in Eq. (3) is related to the gas mass flux

$\Delta q=\frac{Q}{\rho \mathrm{Au}} \frac{x_{2}-x_{1}}{L}$.

$Q$ is the total heating power, $A$ is the cross section area of the capillary, and $L$ its length. An equal distribution of the heating along the capillary is assumed. The term $\Delta q_{u}$ is an additional momentum source, which can be used to model wall friction. In the following, however, wall friction is neglected and the study focuses on heating effects.

The Eqs. 1-3 can be solved analytically (see Supp. Mat. S.5). To determine the flow for the entire length, at the tube's end sonic gas flow is assumed as a realistic boundary condition. Note that the heating changes the speed of sound and thereby the flow conditions. The inflow of the straight part is connected with an adiabatic (non-heated) acceleration from atmospheric conditions in the funnel region.

A typical solution for the capillary flow with a net heating power of $Q=16 \mathrm{~W}$ is shown in Figure 2 in comparison to a limiting case, where no heating power is taken into account. The limiting case $Q=0 \mathrm{~W}$ is shown for comparison, and is discussed further down. It offers an insight into the influence of the heating on the gas flow.

The flow in the funnel $(x<0)$ accelerates due to the changing cross section. This adiabatic acceleration is accompanied by a corresponding pressure drop and cooling before entering the straight part $(x \geq 0)$, where the heating is applied. For the 
Inlet and capillary profile

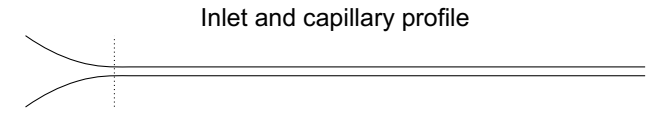

\section{a}

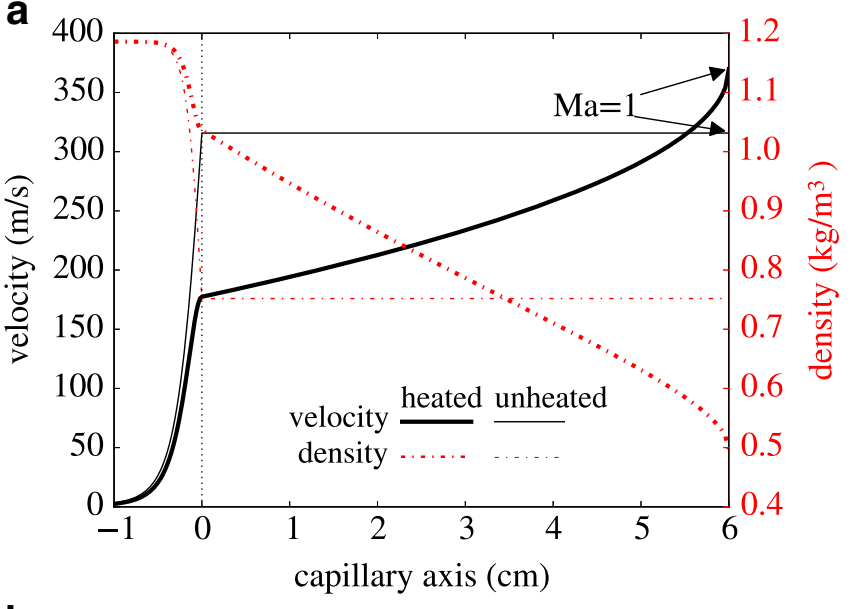

b

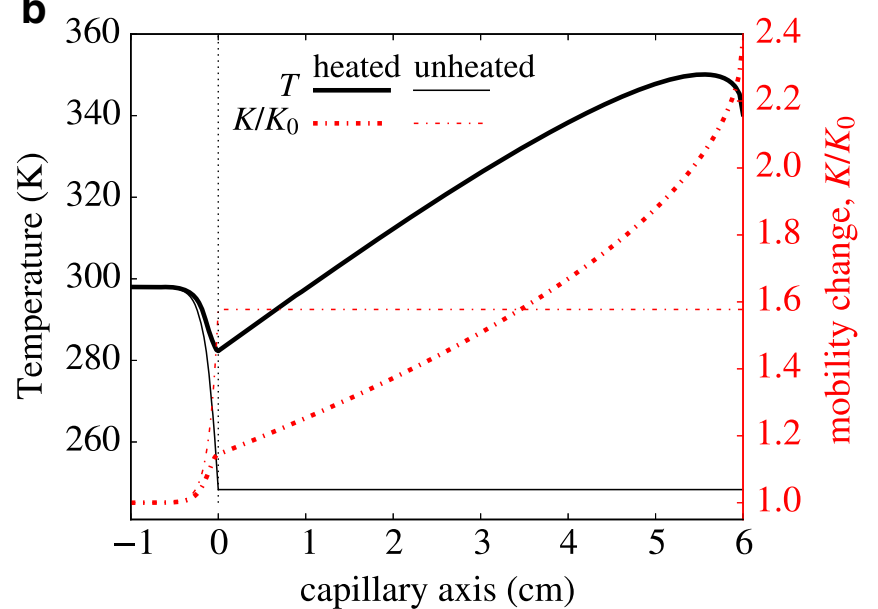

Figure 2. Heated flow $(Q=16 \mathrm{~W})$ and unheated flow $(Q=0 \mathrm{~W})$ through a $6 \mathrm{~cm}$ capillary. The connection of funnel and straight capillary is marked with the broken line at $x=0 \mathrm{~cm}$. (a) Flow velocity (black) and density (red) along the capillary axis. (b) Temperature (black) and mobility change (red). In the idealized unheated limiting case, all quantities are constant over the full length of the straight part, see text. In the heated capillary the velocity and mobility rise dramatically, while the density (pressure) drops. In both cases, the flow is sonic at the end, $\mathrm{Ma}=u / c=1$ with different speeds of sound $c$ due to the heating. Towards the end of the capillary, the temperature drops due to strong compressibility effect close to sonic conditions

heating of $16 \mathrm{~W}$, the flow strongly accelerates within the capillary, from 180 to $350 \mathrm{~m} / \mathrm{s}$, whereas without heating, the flow velocity is constant at the speed of sound (Figure 2a).

The temperature of the heated gas flow rises by $60 \mathrm{~K}$ (see Figure $2 \mathrm{~b}$ ). Interestingly, at the end of the transfer tube, the temperature of the gas flow drops, despite further heating. This counter-intuitive behavior is well-known for near-sonic flows, where cooling induced by the gas expansion overcompensates the rise of internal energy [22].
In comparison, in the unheated case, the full acceleration happens within the funnel, and a constant flow at sonic conditions is found in the straight part. This is an idealization, since even for no electric heating, a heat flux into the gas is expected. For a well isolated capillary, even the wall friction should be considered, leading to a non-constant flow along the capillary length (see [21] and Supp. Mat. S.3).

Finally, the ion mobility can be extracted from the calculated gas state. Being dependent on both pressure and temperature, it changes with the heating of the gas correspondingly, increasing by a factor of 2 along the axis in the example of Figure $2 \mathrm{~b}$. This strong change in mobility is a direct indication of strongly modified transport characteristics upon heating, affecting the transport in the electric field as well as the diffusion (see Sec. 2.4.1-Diffusion). Similarly, the transport via drag force is depending on the flow velocity, and finally as outlined initially, the laminar or turbulent state of the flow will critically depend on the change of the parameter calculated here. As a consequence of the dramatic, non-trivial gradients of the gas properties along the tube, treating the ion transport through a heated capillary clearly requires considering the local properties of the flow.

Furthermore, while the gas flow is modeled by a onedimensional approximation, the ion motion will be simulated fully three-dimensional over the entire volume of the conduit. The chosen approach, described in Supp. Mat. S.7, combines a rotational symmetric profile with the one-dimensional description of the flow, to take into account the presence of the capillary walls. The profile depends on the nature of the flow. In the laminar case, the analytical solution of a parabolic profile is applied, and in the turbulent case, a well-established heuristic profile is used. This one-dimensional description of the flow is furthermore useful to estimate the pressure in the first vacuum chamber connected to the API. Two limiting models can be applied, which are presented in Supp. Mat. S.6.

Turbulent and Laminar Gas Flow While the magnitude of the Reynolds-number suggests the occurrence of turbulent flow, the inflow from a resting gas through the slowly converging funnel inlet is initially laminar. The development of turbulence requires a certain distance or time, which depends on the magnitude of the Reynolds-number and may be promoted by disturbances in the initial inflow.

Particularly, if the inflow is strongly disturbed, as for instance by a sharp conduit edge, turbulence will rapidly develop and the entire flow will be turbulent. A proper prediction of the transition to turbulence is delicate [27] and demands a detailed simulation. Here, a simple approach is followed instead.

If the inflow is reasonably smooth, a transition length estimate is given by

$l_{t}=\alpha_{t} \times d / \mathrm{Re}$.

with a literature value for the empirical parameter $\alpha_{t}=500000$. ([28], p. 245). 
For simplicity, the transition is treated to occur instantaneously at $x=l_{t}$, and we use the Reynolds number at the inflow for calculating the transition length. If $l_{t}>L$, the flow is treated as laminar throughout the entire capillary of length $L$.

It should be noted that this is a very rough, empiric estimate. Generally, the occurrence of this transition depends on the inflow, environmental conditions, and even on details of the experimental geometry. In particular, the Reynoldsnumber in Eq. 5 changes along the conduit length due to its dependencies, which are all functions of the state of the flowing gas.

Moreover, the acceleration of the flow by the heating has a direct suppressing effect on the occurrence of turbulence. The change of the Reynolds number and the acceleration along the tube are closely linked and therefore their individual influence is difficult to distinguish. The simple modeling used here (Eq. 5 ) is based solely on considering the Reynolds-number while the acceleration is not explicitly included.

This is justified because both influences work towards the same effect that is delayed turbulence, so that fitting the parameter $\alpha_{t}$ covers both. Hence, no separate treatment is attempted. Again, a turbulence resolving gas dynamic simulation would be necessary for a detailed investigation of this effect.

The distinction of laminar and turbulent flow is pivotal for the description of the ion transport. Depending on the state of the gas flow, we will encounter different velocity profiles, different wall friction, as well as significantly changed diffusion, which has a profound effect on losses and transmission.

\section{Simulation of the Ion Transport}

Based on the gas flow along the transfer conduit as determined in Sec. 2.3, we now approach the simulation of the ion transport. This includes the treatment of laminar and turbulent flow regions as well as the transition between them. To account for the effect of space-charge repulsion, a large number of mutually interactive ions (in the order of $10^{5}$ ) are simulated moving through the conduit alongside the flowing gas. External fields, in contrast, are expected to play a role only at the inlet and exit of the capillary, but not within the metal tube and are therefore neglected. Nonetheless, the pre-acceleration of the inlet region must be considered for the initial distribution of ions entered into the simulation [8]. Further, the generation of the ion cloud by an electrospray demands the incorporation of droplet desolvation. In the following, the simulation methodology is described in detail, and the basic cases of a laminar and turbulent diffusion are analyzed.

Simulation Methodology For the ion transport simulation, we assume that the droplet desolvation process is complete and all charges are present as molecular ions. The ions are modeled as point particles, with fixed charge, mass, and mobility. Constant time steps of $\Delta t=3 \times 10^{-7} \mathrm{~s}$ are used throughout the simulation. The position of each ion $\boldsymbol{x}_{p}$ is updated in every time step $\Delta t=t_{n+1}-t_{n}$ from the old positions by

$\boldsymbol{x}_{p}\left(t_{n+1}\right)=\boldsymbol{x}_{p}\left(t_{n}\right)+\Delta t \boldsymbol{v}_{i}+\Delta \boldsymbol{x}_{\mathrm{rand}}$

Here, $\boldsymbol{v}_{i}$ is a deterministic velocity of the ion, and $\Delta \boldsymbol{x}_{\text {rand }}$ a random displacement to model diffusion. The ion velocity is given by

$\boldsymbol{v}_{i}=\boldsymbol{v}_{g}+\boldsymbol{E}_{\mathrm{sc}} K$

Here, $\boldsymbol{v}_{g}$ is the velocity of the gas, $\boldsymbol{E}_{\mathrm{sc}}$ is the electric field created by the space charge of the other ions, and $K$ is the ion mobility, which depends on gas pressure $p$ and temperature $T$ as

$K=K_{0} \frac{T}{T_{0}} \frac{p_{0}}{p}$

where $K_{0}$ is the value for the reference conditions $T_{0}=$ $273.15 \mathrm{~K}, p_{0}=1013.25 \mathrm{hPa}$. This explicit expression for the velocity neglects an acceleration phase, because the ions are following the motion of the gas near instantaneously as approximated initially (see Sec. 2.1).

Particles are introduced at random with a Gaussian distribution of selectable width at the position of desolvation $l_{d}$, i.e., where the gas phase ions are generated from the droplet (see Figure 1). If an ion is found outside the capillary radius after the time step, it is considered lost. This implies that all ions vanish when hitting the metal wall. Measurements with low energy ions on metal surfaces suggest that this is a valid assumption [8], while the situation on insulation surfaces may be different. Finally, if an ion is found beyond the end of the capillary, it is counted as transmitted. Both lost and transmitted ions are reintroduced. The simulation runs until a steady state is reached, i.e., when the loss and transmission currents are constant.

Space-Charge Interaction The mutual interaction of the ions, i.e., the space-charge field $\boldsymbol{E}_{\mathrm{sc}}$, is an essential part of the current study, since high-charge densities can lead to a Coulomb-blockade-dominated transport. The particle-particle interaction is treated via a fast multipole method [29], implemented by Yokota [30]. A direct evaluation of the interaction between the $N$ particles would lead to $N(N-1) / 2$ evaluations, which is not feasible for large $N$. The fast multipole approach reduces the numerical effort so that in principle, the full number of ions in the capillary could be simulated on a computing cluster. To further save processing time, the potential created by each ion is scaled by a factor, thereby reducing the number of simulated ions by that same factor, permitting the use of a regular PC. Note that the force acting on the particle is still proportional to its charge. All simulations were performed with 40,000 ions; convergence was tested with 80,000 ions. Ion currents are calculated including this multiplier. 
Diffusion Diffusion is modeled by random jumps $\Delta \boldsymbol{x}_{\text {rand }}$ in the $x$-, $y$-, and $z$-direction. Using the isotropy of diffusion, a common jump distance $\Delta l$ is used and one can write:

$\Delta \boldsymbol{x}_{\mathrm{rand}}= \pm \Delta l \cdot \boldsymbol{e}_{x}+ \pm \Delta l \cdot \boldsymbol{e}_{y}+ \pm \Delta l \cdot \boldsymbol{e}_{z}$,

where $\boldsymbol{e}_{x}, \boldsymbol{e}_{y}$, and $\boldsymbol{e}_{z}$ are the unit vectors in the $x$-, $y$-, and $z$ direction, respectively. The jump distance $\Delta l$ is chosen such that the correct diffusivity is created [31], i.e., the average distribution of particles will behave as described by the diffusion law, with the diffusion constant $D$ defined as

$D=(\Delta l)^{2} /(2 \Delta t)$.

The diffusion constant is composed of two parts

$D=D_{\text {brown }}+D_{\text {turb }}$

reflecting the random movement caused by molecular collisions $\left(D_{\text {brown }}\right)$ and the quasi random displacement modeling the turbulence $\left(D_{\text {turb }}\right)$.

The first contribution is Brownian diffusion. It is connected to the ion mobility $K$ via the Einstein relation, as described in [32], by the following relation:

$D_{\text {brown }}=K \times \frac{\mathrm{kt}}{e}$,

with the Boltzmann constant $k$, temperature $T$, and charge of the ion $e$.

The turbulent diffusion is, following Hinze [33], assumed to be

$D_{\text {turb }}=0.02 \bar{u} d \sqrt{\lambda}$,

This heuristic law for turbulent pipe flows describes the effect of turbulence by quasi random and isotropic velocity fluctuations. It matches well with the fact that turbulence strongly increases mixing, quickly leading to an isotropic distribution. Here $\bar{u}$ is the mean velocity, set to the local gas velocity $\bar{u}=\left|v_{g}\right|, d$ the capillary diameter, and $\lambda$ is the wall friction coefficient.

The wall friction coefficient, as given in [33], being solely dependent on the Reynolds number, is empirically described by

$\sqrt{\frac{8}{\lambda}}=2.44 \ln \left(\frac{\lambda}{32} \operatorname{Re}\right)+2.0$.

This implicit description for $\lambda$ at every position along the capillary can be quickly solved numerically by fixed-point iteration.
Basic Configurations Based on the simulation methodology introduced above, we can now analyze several basic cases, distinguished by the following:

The state of the flow: either laminar or fully turbulent Heating of the tube: either heated or not heated Space charge: switched on or off

Six combinations comprising the set of these basic regimes were simulated for an ion current of $5 \mathrm{nA}$ in a truncated, broad Gaussian beam starting distribution with a standard deviation of $\sigma=d / 4$ and the heating set to $Q=16 \mathrm{~W}$. Figure 3 shows the electric current through the capillary as a function of position. For all cases, the current drops along the axis with great variations of slope, which represents different loss rates. The strongest distinction is found between the turbulent and laminar flow conditions. In the turbulent cases, the strongly enhanced diffusion dominates all other influences. For fully laminar flow, space charge makes a significant impact, whereas heating has only little effect on the ion transport.

To explain this small impact of heating despite the strongly changed transport properties, let us consider the transport of a single particle by space charge. The velocity in axial direction $u$ is dominated by the gas flow and can be written as a traveled distance $\Delta x$ over a passed time interval $\Delta t$

$\Delta x / \Delta t=u$.

The radial transport $\Delta r$ can equivalently be expressed by the radial electric field $E_{r}$ and the mobility $K$ by substituting the ideal gas law at (1) and the mass conservation at (2):

$\Delta r / \Delta t=E_{r} K=E_{r} K_{0} \frac{T}{T_{0}} \frac{p_{0}}{p} \stackrel{1}{=} E_{r} K_{0} \frac{\rho_{0}}{\rho} \stackrel{2}{=} \frac{E_{r} K_{0}}{u_{0}} u$,

depending on the mobility and axial velocity as well. The values with the subscript 0 are reference values at the start of the straight part of the capillary. Thus from Eqs. (14) and (15), it follows a relation for the ratio of axial and radial displacement

$\Delta r / \Delta x=\frac{E_{r} K_{0}}{u_{0}}$

in which a strong increase in mobility is largely canceled by the change in velocity due to heating. This consideration is interesting as it shows that heating influences the ion transport in several ways, which, however, nearly cancel each other for the overall transmission. Space charge, present as $E_{r}$ in Eq. 16, will thus gain influence for higher currents. A similar argument can be made for a friction-induced flow change, since the conservation of mass applies in the same way. This at least partly explains why the simulation reproduces experimental data well, in spite of neglecting wall friction in the gas flow, as detailed later. 


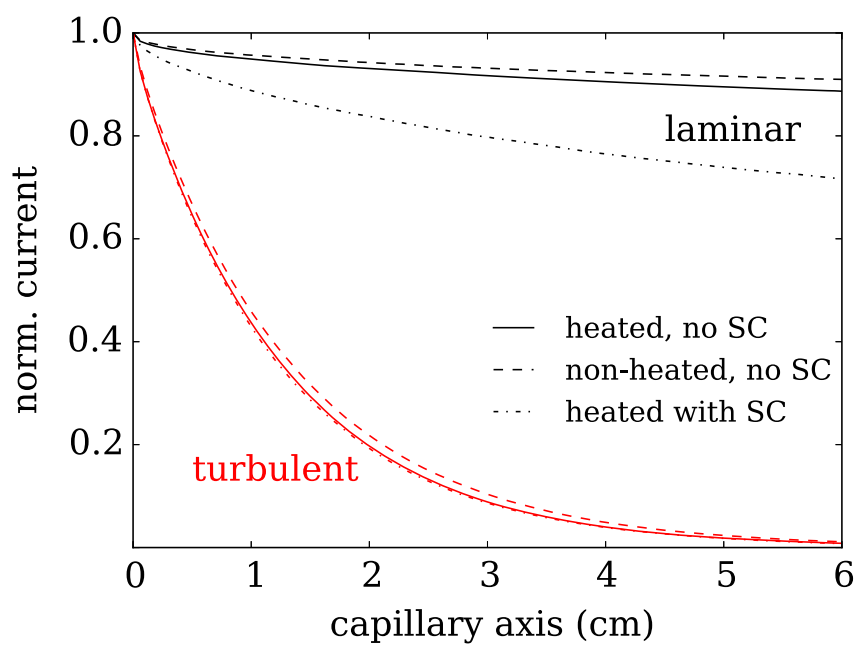

Figure 3. Simulated (normalized) ion current flowing in the capillary. The current is reduced due to losses, when ions collide with the wall. Basic cases are considered fully laminar and fully turbulent, heated and unheated, and with or without space charge (SC)

Still, the heating has an influence by causing thermal choking due to modifying the inlet velocity $v_{0}$ to fulfill sonic condition at the end of the capillary. In the real experiment, this change in inlet velocity might have consequences for the behavior of the capillary inlet. Specifically, lower gas velocities in the inlet due to choking can lead to a reduced collimation of the initial ion cloud, a suppression of the space charge at the spray-needle tip due to fast removal of generated ions, and changes in the flight length of droplets [8].

Overall, the significant changes of the flow due to heating lead to a surprisingly small effect on the ion transport itself, whereas the turbulence has a dramatic effect. Furthermore, the influence of space charge is only observable for laminar flows, while the estimation of the Reynolds numbers for the gas flow in the transfer tubes suggests turbulence, yet it will scale with higher currents.

It thus becomes clear that in order to be able to reproduce the behavior of realistic vacuum transfer interfaces not only the ion transport in the flowing, heated gas has to be considered but also the interaction with the surrounding such as droplet desolvation, inflow characteristics, or laminar/turbulent transition.

\section{Modeling the Experiment}

Conventional electrospray capillary interfaces typically feature long, narrow capillaries and are thus found to operate at low transmission [5, 12, 34-37]. This behavior can be explained with the observation made for the basic configurations, calculated in the previous section. They show that high losses are caused by turbulent diffusion, which generally occurs, as suggested by the high Reynolds numbers of the typical transfer capillary. However, to understand the recent observation of very high, even up to unity, ion current transmission in nanospray interfaces featuring short wide capillaries $[8,9,38]$, these simple cases are not sufficient.
Therefore, the presented ion transport simulation describing gas flow, space charge, and diffusion, further needs to account for the influence of phenomena such as turbulent/ laminar transition, droplet desolvation, and inflow characteristics. We thus extended the simulations to encompass a simulated, heated gas flow with a laminar inflow, which can transit to turbulence. Further, the ion transport is simulated in this flow, starting with an ion beam generated at the point of droplet desolvation, which can be within the capillary. Towards reproducing the experimental data with this extended model, the hitherto open modeling parameters need to be adjusted. Those are (1) the ion inlet distribution given by the Gaussian width of the injected ion beam $\sigma$, (2) the point of droplet desolvation $l_{d}$, and (3) the position of the laminar turbulent transition $l_{t}$, defined through Eq. 5 by the coefficient $\alpha_{t}$ (see Figure 1). With our model, we aim to reproduce experimental observations, which are the transmission as a function of heating (Figure 4) and the ion current transport characteristics $I_{\text {trans }}\left(I_{\mathrm{em}}\right)$ (Figure 5).

Laminar-Turbulent Transition Figure $4 \mathrm{~b}$ shows the transmission measured for two different emitted currents as a function of temperature. Between 100 and $150{ }^{\circ} \mathrm{C}$, a characteristic rise of the transmission with a slow decrease following after the maximum at $155^{\circ} \mathrm{C}$ is observed. A transition from turbulent to laminar upon heating can explain this temperature dependence, associating the low transmission with a turbulent state. The modeling of the location $l_{t}$ according to Eq. 5 is illustrated in Figure 4 , top, showing the point of turbulent transition as a function of the temperature and indicating the flow regimes. For any given temperature, the flow in the capillary is laminar on inflow and becomes turbulent after traveling the distance $l_{t}$. Above a certain temperature, $l_{t}$ will be larger than the capillary length and hence the flow will be laminar throughout the entire capillary. Under this circumstance low losses can be achieved due to the much lower diffusion in the laminar flow. The reduction of the transmission for higher temperatures is explained by thermal chocking, see Sec. 3.1.

With the Reynolds number determined by the calculated flow, only the coefficient $\alpha_{t}$ can be adjusted so that $l_{t}$ equals the capillary length at the transmission maximum. This fit gives $\alpha_{t}=770000$, which is in reasonable agreement with the literature value in [28], given the empiric nature of the relation used. These resulting modeled temperature characteristics are shown alongside the measurements in Figure 4, bottom.

While implementing a turbulent transmission in the ion transport model reproduces the non-trivial temperature dependence, the exact temperature of the turbulent/laminar transition is strongly bound to geometry and manufacturing quality of the capillary. In particular, the use of a funnel inlet is crucial to guaranty an undisturbed laminar inflow. For example, extensive polishing can remove the transition altogether, and the high transmission characteristic of laminar transport is seen already at room temperature. 

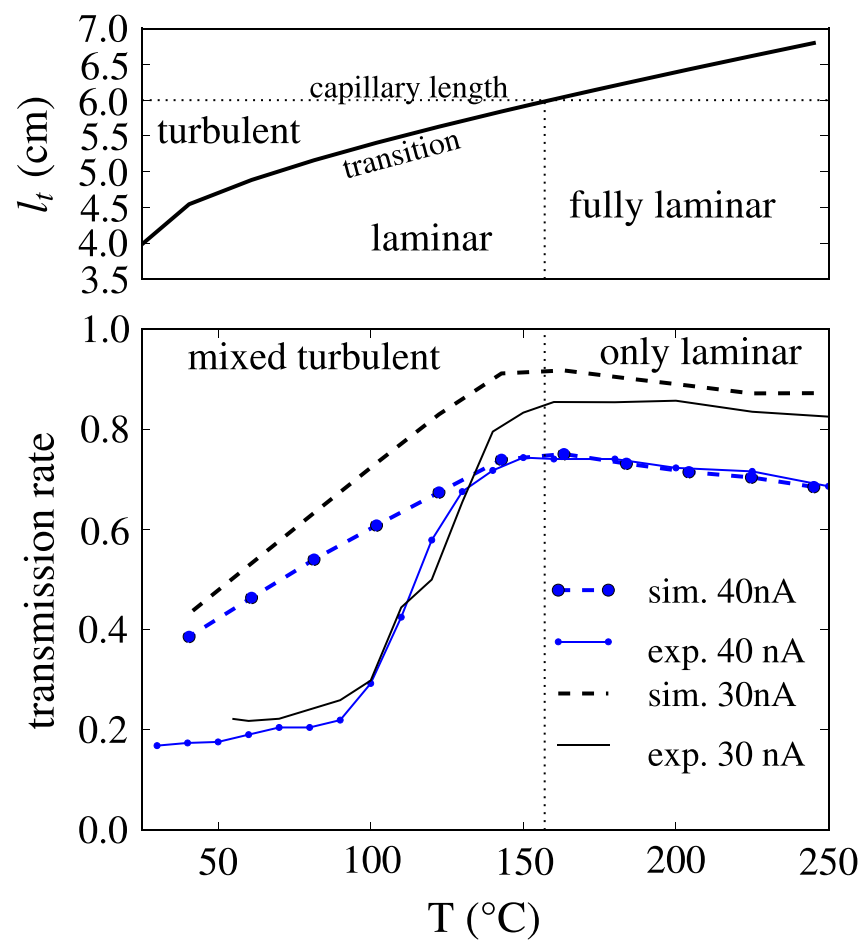

Figure 4. Temperature dependence of the transmission measured and simulated for two different currents (bottom) and temperature dependence of the position of the laminar-toturbulent transition within the capillary (top). For a capillary length of $6 \mathrm{~cm}$, the turbulent transition is located within the capillary for temperatures below $155^{\circ} \mathrm{C}$, and the full capillary is laminar above this temperature. The increased thermal choking of the fully laminar state has a (mild) adverse effect on the transmission. These two effects explain the non-trivial transmission characteristics

Droplet Desolvation and Ion Beam Shape Generally, heating of the gas flow is intended to yield a good desolvation and enhance ion formation, which is confirmed experimentally [2, 12]. Therefore, we want to contrast the turbulence interpretation of the observed temperature characteristics with the alternative interpretation that the temperature dependence is due stemming from droplet desolvation. By accelerating the droplet desolvation, the heating of the gas flow reduces the droplet life time and thus its transport length in the capillary. As a consequence, the transport length of the gas phase ions increases. Lighter ions, however, are more susceptible to space charge and diffusion, so a longer transport length leads to higher losses (see Supp. Mat. S.8 and [7]). By this rationale, the overall transmission should decrease steadily with heating. Indeed, both in the experiments and the simulation, this slow drop of transmission for high temperatures is observed [12] at temperatures beyond the sudden strong increase attributed to the transition to fully laminar flow (Figure 4, bottom). However, the slow decrease in the simulation arises solely from the slower inflow by the thermal chocking and captures the decrease in transition well, whereas the droplet flight length is kept constant.
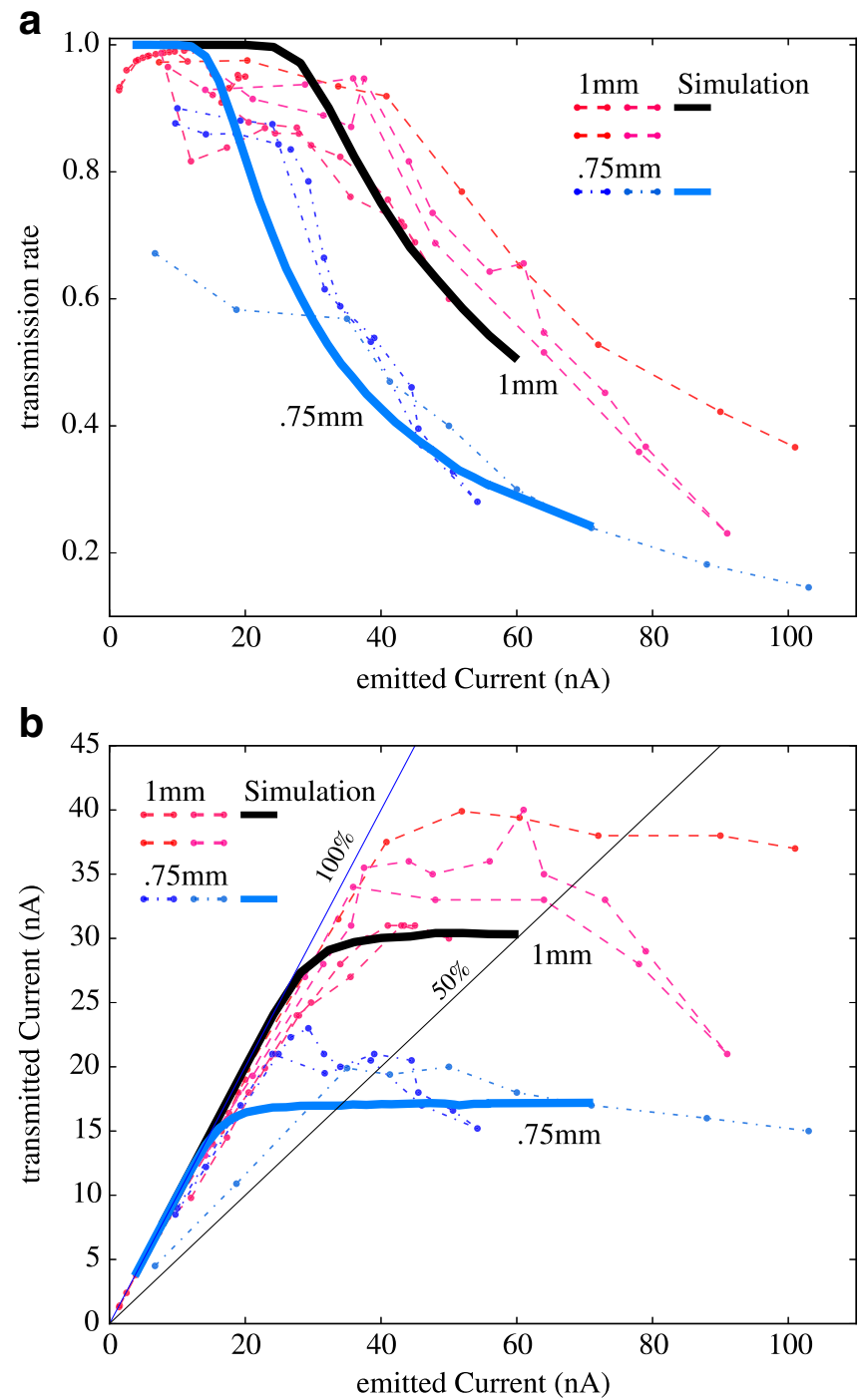

Figure 5. The transmission rate (a) and transmitted current (b) as a function of the emitted current for a conduit diameter of $1 \mathrm{~mm}$ and $0.75 \mathrm{~mm}$, for several measurement campaigns compared with the simulation. Space charge-driven blocking becomes visible at 32 and $17 \mathrm{nA}$ limiting the transmitted current. The measurement runs show considerable variation

While the occurrence of the laminar/turbulent transition solely depends on the gas flow and is thus unaffected by any injected ions or charged droplets, the level of transmission and losses is strongly influenced by the ion creation position $l_{d}$. For fitting the model to the observed temperature characteristics, the droplet desolvation position was chosen such that the laminar flow part of the $40 \mathrm{nA}$ curve matches experiment and simulation, since in this regime (without the empiric turbulent diffusion), the ion transport is more reliably described. The position found is $l_{d}=1.6 \mathrm{~cm}$, which is reasonable, considering the capillaries geometry (see Supp. Mat. S.2). Further, the same value of $l_{d}$ is used for the $30 \mathrm{nA}$ case and also for all following simulations, producing results of quantitative accuracy.

The ion creation by droplet desolvation is modeled in radial direction as a sharp Gaussian distribution of $\sigma=R / 20$. The 
sharp distribution is decisive for the initial losses. For a broad starting distribution, losses are found even for a laminar flow and low space charge. A narrow distribution is likely for the funnel-shaped inlet [8]. This is later rationalized by snapshots of the simulation and quantitatively discussed in Sec. 3.2.

Transmission Characteristic/Space Charge Figure 5 shows several measured transmission characteristics for two funnelinlet transfer capillaries of $1-$ and $0.75-\mathrm{mm}$ diameters in two different visualizations, first transmittance $\left(T=I_{\text {trans }} / I_{\mathrm{em}}\right)$ as function of emission current $\left(T\left(I_{\mathrm{em}}\right)\right.$, (Figure $\left.5 \mathrm{a}\right)$ and second transmission current as function of emission current $\left(I_{\text {trans }}\left(I_{\mathrm{em}}\right)\right.$, (Figure 5b) [8]. They are characterized by a close to $100 \%$ transmission for low emission currents, which levels off for high emission currents to a constant maximum current at $32 \mathrm{nA}$ for the $1.0 \mathrm{~mm}$ capillary and $17 \mathrm{nA}$ for the $0.75 \mathrm{~mm}$ capillary.

For the simulations, the heating is set to optimal values of $7 \mathrm{~W}$ and $3.9 \mathrm{~W}$ for the 1 and $0.75 \mathrm{~mm}$ capillaries respectively, so that laminar flow is present in the entire capillary, corresponding to the maximum transmission visible in Figure 4. The simulated characteristics then reproduce the two major experimental observations quantitatively: First, starting from about $30 \mathrm{nA}$, a saturation of the transmitted current for the $1 \mathrm{~mm}$ diameter capillary, and at about $17 \mathrm{nA}$ for the $0.75-\mathrm{mm}$-diameter capillary is found, see Figure $5 \mathrm{~b}$. In the simulation, it can be identified as a space-charge blocking. Second, the transmission for both capillaries is initially close to $100 \%$. The later drop is equivalent to the saturation of the transmission current.

\section{Discussion}

In order to summarize the achievements of the presented simulation and shine a critical light on its limits, this section first presents a reflexion on the ion motion from a microscopical point of view and then discusses the influence of the used parameters by presenting their limitation and implications for the design of API.

\section{Microscopic View of the Ion Motion}

To understand the ion motion, loss mechanisms and the influence of the heating, snapshots of the simulation for different conditions were extracted. One situation for a low $\left(3 \mathrm{~W}, 81{ }^{\circ} \mathrm{C}\right)$ and one for a high $\left(7 \mathrm{~W}, 163{ }^{\circ} \mathrm{C}\right)$ heating power are presented in Figure 6, both for a capillary diameter $d=1 \mathrm{~mm}$ and an input current of $40 \mathrm{nA}$, where space-charge losses become important. The gas flows from left to right. The ions are inserted close to the axis (at $l_{d}=0$ used for this visualization) but quickly expand due to space charge and diffusion. The expansion rate is higher for the $7 \mathrm{~W}$ case when the gas velocity is lower due to thermal choking, resulting in higher losses closer to the inlet as in the $3 \mathrm{~W}$ case, visible in the loss current plot at 2-3 cm (Figure 6c).

For $3 \mathrm{~W}$, our modeling for the turbulence transition length is shorter than the capillary. The high turbulent diffusion sets in at about $x \approx 5.2 \mathrm{~cm}$, marked by the red, broken line. This leads to a high loss current at the end of the capillary. Here, we can clearly distinguish the two effects that contribute to the temperature dependence of the transmission. One, the suppression of turbulence with heating strongly increases the transmission. Two, the decrease in inflow velocity due to thermal choking decreases it. Thus, as soon as the turbulence is fully suppressed, the adverse effect of the heating becomes visible and reduces transmission again, which defines an optimal heating as the lowest value to suppress turbulence. To compare the relative importance of the different effects, effective ion fluxes attributed to each are shown in Supp. Mat. S.9.

The velocity for the space-charge blocking regime is quite high, velocities of the order of $1 \mathrm{~m} / \mathrm{s}$ occur due to fields of order $\mathrm{kV} / \mathrm{m}$ at the beginning, suggesting a non-linear mobility law to describe the velocity of the ions. However, the space-charge effects quickly drops in this regime, which likely explains the good agreement of the results with the measurements and justifies a posteriori the use of a linear law. A non-linear law most likely changes the position of the maximum of Figure $6 \mathrm{c}$, but the effect on the total loss is expected to be small.

For emitted currents below the space-charge blockade, the space-charge-driven expansion is not sufficient to expand the ion cloud from the initial narrow distribution to the wall during the passage through the capillary, which is the condition needed for unit transmission. This narrow distribution is created by the collimation effect of the funnel-shaped inlet due to the converging gas flow [8].

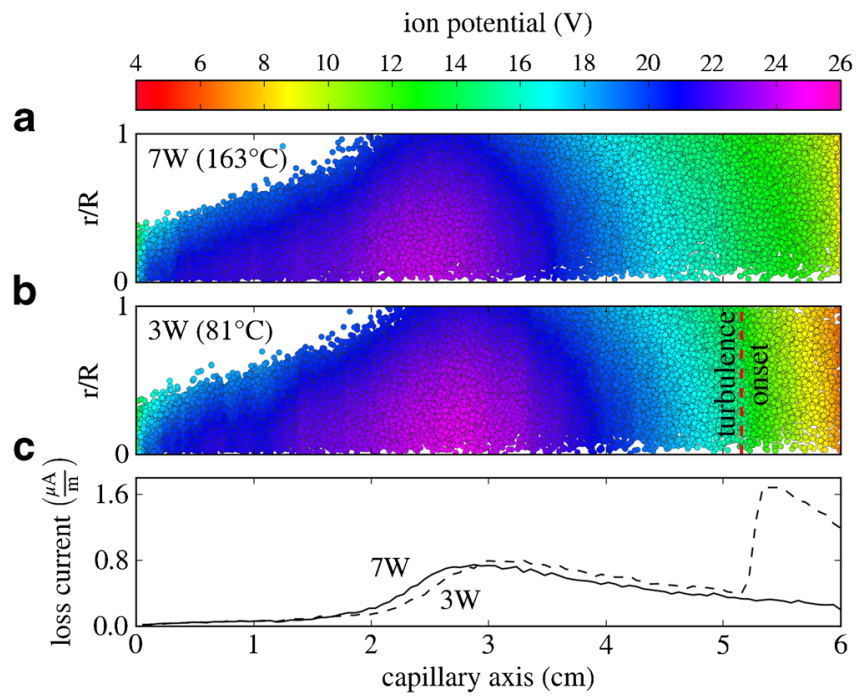

Figure 6. Snapshots of two runs for low $(3 \mathrm{~W})$ and high $(7 \mathrm{~W})$ heating and loss current. $(\mathbf{a}, \mathbf{b})$ Plots show the radial position of the particles from the axis as a function of the position along the capillary. The color scale represents the ion potential. While in the $7 \mathrm{~W}$ case, the heating is sufficient to suppress the turbulence for the full capillary length; in the $3 \mathrm{~W}$ case, the transition to turbulence still happens within the capillary. (c) The first maximum in the spatial resolved loss rate is space chargedriven and shifts due to chocking. The second maximum in the $3 \mathrm{~W}$ simulation arises from turbulence and leads to high losses 


\section{Modeling Parameters and Experimental Design}

The modeling needed to reproduce the experimental observations provides physical insight and important indications for the design of ESI interfaces. The droplet transport, the initial ion distribution, and the occurrence of turbulence are found to be essential. The modeling developed here depends on three parameters, the droplet transport length $l_{d}$, the initial ion distribution width $\sigma$, and the factor $\alpha_{t}$ used in the description of turbulent transition length $l_{t}$.

The complex process of droplet desolvation is condensed into the ion creation length $l_{d}$, which assumes that after a transport length, the complete desolvation cascade is highly rapid [25]. This avoids the need for information on the initial droplet size, which is hard to obtain. The value found for $l_{d}$ works for all experimental observations, and the model is further justified by an estimate based on the gas flow in the funnel region (Supp. Mat. S.2). For significant changes of solvent or emitter, this parameter would need to be adjusted.

The droplet transport length $l_{d}$ and the ion distribution width $\sigma$ are not fully independent, since a larger $l_{d}$ can be compensated by a broader distribution mimicking the space-chargedriven expansion. However, a finite $l_{d}$ is crucial to reproduce the transmission rate, showing that droplet properties have a severe effect on the transmission, which is discussed further in Supp. Mat. S.8.

The influence of the distribution width $\sigma$ on the transmission rate, for different emitted currents, and a given droplet desolvation length is represented in Figure 7. In all cases, a more narrow ion distribution leads to higher transmission rates by increasing the average time for an ion to reach the capillary wall. It shows that a sharp distribution $(1 / \sigma \gg 1)$ is the first condition to achieve unit transmission in the capillary.

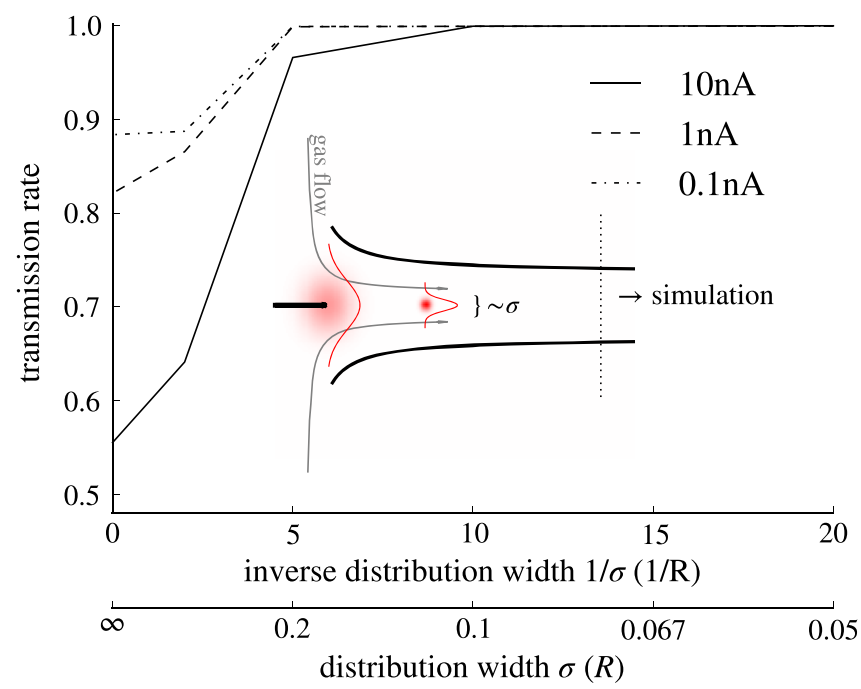

Figure 7. The ion inlet distribution influences the transmission. It is modeled by a Gaussian distribution with $\sigma$ as the standard deviation; for $R / \sigma=0$, the distribution is flat. For a high transmission at high currents, a sharp distribution is vital. The funnel shape of the capillary inlet leads to a collimation of the ion cloud by the flow, as discussed in [8]
The second condition is a laminar gas flow. To qualitatively reproduce the behavior of the transmission changing with heating (see Figure 4), a turbulence transition has to be introduced into our description. The factor $\alpha_{t}$, used in the transition length estimate, sets the lowest heating for which the capillary flow is fully laminar and has thereby a strong impact on the transmission rate.

The identified, decisive effects can be influenced by design and operation of the ESI. The funnel inlet has two impacts. First, it collimates the droplets and thereby the ions, as was already identified in [8] and schematically depicted in Figure 7. It further creates a smooth inflow, helping to delay the turbulent transition. This transition is furthermore influenced by heating. Our description suggests that a fully laminar flow can be reached by heating in the experiment.

Since the turbulent transition is a complex process, which depends sensitively on details of the flow field and disturbance level, this effect is expected to be different for different capillaries, and might even change for different measurement campaigns when barely noticeable details change. While only laminar flow can explain the good performances presented in [8], turbulence cannot be ruled out at the given Reynolds numbers, and it is important to notice that only turbulence is able to explain some experimental observations. In general, laminar flow at lowest possible heating will lead to the best transmission (see Supp. Mat. S.8). Consequently, reducing disturbances by a better manufacturing of capillaries is helpful and better geometries can probably be found.

The droplet desolvation length $l_{d}$ is a very important parameter because of its strong impact on the transmission rate (see Supp. Mat. S.2). It should change with the droplet size or a different emitter position. The first is influenced by different factors like the solvent flow rate, [25] while the emitter position can be tuned. Thus, the latter seems a good way to find an optimal transmission while avoiding the passage of droplets. For the description of other experiments, especially if different ion sources and flow rates are used, a more complex ion creation scheme might be needed in the simulation.

\section{Summary and Conclusions}

By a combined modeling of the gas flow and the ion movement under the influence of space charge, we found a consistent, quantitative picture of the ion transport through a heated capillary of an atmospheric pressure interface. The characteristics of our model reproduce the behavior observed for heated capillaries with funnel-shaped inlets, and we are able to study many aspects of the fundamental behavior of the ion transport in capillaries.

The one dimensional description of the gas flow with velocity profiles is, in a first approach, largely sufficient to describe the gas transport through the capillary, quantitatively matching the measured pumping pressures. In particular, we show that heating has a tremendous effect on the properties of the gas flow, as velocity, density, and mobility are significantly 
affected. Also, it leads to thermal choking of the flow, which accelerates the flow but at the same time reduces the transported amount of gas. The properties of the gas flow, in particular the sufficiently large Reynolds number, suggest the presence of turbulence. We find a simple approximation for the laminar to turbulent transition, whose properties match the dimensions of the problem and the observations made.

With this model, we were able to describe under which conditions a high transmission of up to $100 \%$ can be achieved. Most importantly, unity transmission is only possible for laminar flows, and the injected ions have to be collimated close to the axis of the capillary. Those conditions can be achieved by a low flow electrospray source, in conjunction with a funnelshaped inlet. The funnel geometry has a second crucial purpose that is the promotion of a smooth, laminar inflow delaying the turbulent transition. Indeed, wind tunnels use similar converging nozzles to laminarize the flow [28].

The turbulent transition can be delayed and eventually suppressed by heating, which allows for the observed high transmission. Above a certain ion current, a space-charge blockade is limiting the maximum transmission current.

While the experimental comparison was done for relatively short and wide capillaries with a smooth funnel-shaped inlet, conclusions for other capillaries can be drawn from our model. Since no efficient initial collimation takes place in capillaries with standard, flat inlets, their initial ion distribution is wide. Further, the inflow will be disturbed yielding turbulence for sufficiently wide capillaries. Ion loss similar to the generic turbulent case is expected, resulting in transmission of a few percent. In a longer capillary, space-charge repulsion has more time to expand the ion cloud, adding to the loss. Here a lower space-charge blocking current is expected. This can be partly compensated by long droplet life time of larger droplets.

A very long capillary can reduce the flow rate beyond conditions needed to support turbulence. However, in this case, the space charge and molecular diffusion are likely to overcompensate the effect and lead to a low transmission.

The picture derived here can also explain recent reports of a second case of unity transmission, where funnel-shaped capillaries, opening towards the vacuum side, were used [9]. In such a geometry, the flow is accelerated throughout the entire capillary, which suppresses turbulence. Due to the diverging geometry, the sonic condition is reached at the start of the capillary, and the further expansion leads to a reduction of density and temperature. This could explain the reported difficulties with droplet desolvation, problems that can be avoided if the gas flow stays longer in contact with the tube wall.

Based on our work, the properties of capillary interfaces can be predicted and optimized. Modeling an unknown interface merely requires the geometry and gas properties. The model of the gas flow alone already yields critical design parameters, such as gas load and state of the gas upon entering the first vacuum chamber. Together with the ion transmission simulation, the net current, transmission, and temperature dependence can be estimated quantitatively.
In the presented model, we treat the inlet region (funnel) and the capillary independently, coupling them by fitting three parameters to make for a consistent picture: the lifetime of the droplets, the shape of the initial ion distribution, and the length of the turbulent transition, all quantities which are extremely difficult to acquire otherwise. However, all values used are close to literature values or reasonable estimates.

Further on, exactly these points bear the potential for a deeper understanding and will be investigated in the future work. This study focused on the straight part of the capillary and neglected external fields. Droplet dynamics as well as the coupling of the flow at the capillary inlet and exit were only discussed on a very simple level. Especially the inlet region can be used to improve collimation and suppress the space-charge limits further by an enhanced extraction and collimation using gas flows tailored for this purpose. Therefore, we work on an extended simulation method to include external fields and droplets within a three-dimensionally modeled gas flow describing turbulence and other disturbances on the correct length and time scales.

\section{Funding}

This work is supported by the Deutsche Forschungsgemeinschaft (DFG) under RE-3774/1-1.

\section{References}

1. Bruins, A.P., Covey, T.R., Henion, J.D.: Ion spray interface for combined liquid chromatography/atmospheric pressure ionization mass spectrometry. Anal. Chem. 59, 2642-2646 (1987)

2. Chowdhury, S.K., Katta, V., Chait, B.T.: An electrospray-ionization mass spectrometer with new features. Rapid Commun. Mass Spectrom. 4, 8187 (1990)

3. Dole, M., Mack, L.L., Hines, R.L., Mobley, R.C., Ferguson, L.D., Alice, M.B.: Molecular beams of macroions. J. Chem. Phys. 49, 2240 (1968)

4. Fenn, J.B., Mann, M., Meng, C.K., Wong, S.F., Whitehouse, C.M.: Electrospray ionization for mass spectrometry of large biomolecules. Science. 246(4926), 64-71 (1989)

5. Page, J.S., Marginean, I., Baker, E.S., Kelly, R.T., Tang, K., Smith, R.D.: Biases in ion transmission through an electrospray ionization-mass spectrometry capillary inlet. J. Am. Soc. Mass Spectrom. 20(12), 2265-2272 (2009)

6. Smith, R.D., Loo, J.A., Edmonds, C.G., Barinaga, C.J., Udseth, H.R.: New developments in biochemical mass-spectrometry - electrospray ionization. Anal. Chem. 62(9), 882-899 (1990) 142

7. Lin, B., Sunner, J.: Ion transport by viscous gas flow through capillaries. J. Am. Soc. Mass Spectrom. 5(10), 873-885 (1994)

8. Pauly, M., Sroka, M., Reiss, J., Rinke, G., Albarghash, A., Vogelgesang, R., Hahne, H., Kuster, B., Sesterhenn, J., Kern, K., Rauschenbach, S.: A hydrodynamically optimized nano-electrospray ionization source and vacuum interface. Analyst. 139, 1856-1867 (2014)

9. Krutchinsky, A.N., Padovan, J.C., Cohen, H., Chait, B.T.: Optimizing electrospray interfaces using slowly diverging conical duct (conduct) electrodes. J. Am. Soc. Mass Spectrom. 26(4), 659-667 (2015)

10. Kim, T., Udseth, H.R., Smith, R.D.: Improved ion transmission from atmospheric pressure to high vacuum using a multicapillary inlet and electrodynamic ion funnel interface. Anal. Chem. 72(20), 5014-5019 (2000)

11. Kim, T., Tang, K.Q., Udseth, H.R., Smith, R.D.: A multicapillary inlet jet disruption electrodynamic ion funnel interface for improved sensitivity 
using atmospheric pressure ion sources. Anal. Chem. 73(17), 4162-4170 (2001)

12. Page, J.S., Kelly, R.T., Tang, K., Smith, R.D.: Ionization and transmission efficiency in an electrospray-ionization mass spectrometry interface. J. Am. Soc. Mass Spectrom. 18(9), 1582-1590 (2007)

13. Poehler, T., Kunte, R., Hoenen, H., Jeschke, P., Wissdorf, W., Brockmann, K.J., Benter, T.: Numerical simulation and experimental validation of the three-dimensional flow field and relative analyte concentration distribution in an atmospheric pressure ion source. J. Am. Soc. Mass Spectrom. 22(11), 2061-2069 (2011)

14. Wissdorf, W., Lorenz, M., Poehler, T., Hoenen, H., Benter, T.: Atmospheric pressure ion source development: experimental validation of simulated ion trajectories within complex flow and electrical fields. J. Am. Soc. Mass Spectrom. 24(10), 1456-1466 (2013)

15. Jurčíček, P., Liu, L., Zou, H.: Numerical simulation of monte carlo ion transport at atmospheric pressure within improved air amplifier geometry. Int. J. Ion Mobil. Spectrom. 17(3), 157-166 (2014)

16. Wissdorf, W., Pohler, L., Klee, S., Mueller, D., Benter, T.: Simulation of ion motion at atmospheric pressure: particle tracing versus electrokinetic flow. J. Am. Soc. Mass Spectrom. 23(2), 397-406 (2012)

17. Mayer, T., Borsdorf, H.: Ion transfer from an atmospheric pressure ion funnel into a mass spectrometer with different interface options: simulation-based optimization of ion transmission efficiency. Rapid Commun. Mass Spectrom. 30(3), 372-378 (2016) RCM-15-0412.R1

18. Zhou, X., Zheng, O.: Following the ions through a mass spectrometer with atmospheric pressure interface: simulation of complete ion trajectories from ion source to mass analyzer. Anal. Chem. 88(14), 7033-7040 (2016)

19. Gimelshein, N., Gimelshein, S., Lilly, T., Moskovets, E.: Numerical modeling of ion transport in a ESI-MS system. J. Am. Soc. Mass Spectrom. 25, 820-831 (2014) arxiv.org pre-print

20. Wißdorf, W., Müller, D., Brachthäuser, Y., Langner, M., Derpmann, V., Klopotowski, S., Polaczek, C., Kersten, H., Brockmann, K., Benter, T.: Gas flow dynamics in inlet capillaries: evidence for non laminar conditions. J. Am. Soc. Mass Spectrom. 27(9), 1550-1563 (2016)

21. Skoblin, M., Chudinov, A., Soulimenkov, I., Brusov, V., Kozlovskiy, V.: Gas flow in the capillary of the atmosphere-to-vacuum interface of mass spectrometers. J. Am. Soc. Mass Spectrom. 28(10), 2132-2142 (2017)

22. Anderson, J.: Modern Compressible Flow. McGraw-Hill, Boston (1990)

23. Davidson, P.A.: Turbulence - an Introduction for Scientists and Engineers. Oxford University Press, New York (2004)
24. Konermann, L., Ahadi, E., Rodriguez, A.D., Vahidi, S.: Unraveling the mechanism of electrospray ionization. Anal. Chem. 85(1), 2-9 (2013)

25. Kebarle, P., Liang, T.: From ions in solution to ions in the gas phase - the mechanism of electrospray mass spectrometry. Anal. Chem. 65(22), 972A-986A (1993)

26. Bartlmä, F.: Gasdynamik der Verbrennung. Springer-Verlag, Wien (1975)

27. Avila, K., Moxey, D., de Lozar, A., Avila, M., Barkley, D., Hof, B.: The onset of turbulence in pipe flow. Science. 333(6039), 192-196 (2011)

28. Prandtl, L., Oswatitsch, K., Wieghardt, K.: Führer durch die Strömungslehre, 9. Auflage. Vieweg+Teubner Verlag, Braunschweig (1993)

29. Greengard, L., Rokhlin, V.: A new version of the fast multipole method for the laplace equation in three dimensions. Acta numerica. 6, 229-269 (1996)

30. Yokota, R., Barba, L.A.: A tuned and scalable fast multipole method as a preeminent algorithm for exascale systems. Int. J. High Perform. Comput. Appl. 26(4), 337-346 (2012)

31. Stephen Berry, R., Rice, S.A., Ross J.: Physical Chemistry, Second Edition. Oxford University Press, Oxford (2000)

32. Mason, E.A., McDaniel, E.W.: Transport Properties of Ions in Gases, vol. 6. Wiley, New York (1988)

33. Hinze, J.O.: Turbulence (Mechanical Engineering Series). McGraw-Hill Inc., US (1975)

34. Manisali, I., Chen, D.D.Y., Schneider, B.B.: Electrospray ionization source geometry for mass spectrometry: past, present, and future. TrAC Trends Anal. Chem. 25(3), 243-256 (2006)

35. Zook, D.R., Bruins, A.P.: On cluster ions, ion transmission, and linear dynamic range limitations in electrospray (ionspray) mass spectrometry. Int. J. Mass Spectrom. Ion Process. 162(1), 129-147 (1997)

36. El-Faramawy, A., Michael Siu, K.W., Thomson, B.A.: Efficiency of nano-electrospray ionization. J. Am. Soc. Mass Spectrom. 16(10), 1702-1707 (2005)

37. Marginean, I., Kelly, R.T., Prior, D.C., LaMarche, B.L., Tang, K., Smith, R.D.: Analytical characterization of the electrospray ion source in the nanoflow regime. Anal. Chem. 80(17), 6573-6579 (2008)

38. Don, K., Gunaratne, D., Prabhakaran, V., Ibrahim, Y.M., Norheim, R.V., Johnson, G.E., Laskin, J.: Design and performance of a high-flux electrospray ionization source for ion soft landing. Analyst. 140(9), 2957-2963 (2015) 